\title{
The Necessity of Staging Laparoscopy/Thoracoscopy in Patients Undergoing Thoracolaparoscopic Oesophagogastric Resections for Malignancy
}

\author{
Mohamed Salah Abdelhamid ${ }^{1, *}$, Ayman Abouleid ${ }^{1}$, Ahmad Mohamed Sadat ${ }^{1}$, Ahmad Hamouda ${ }^{2}$, \\ Amir Nisar ${ }^{2}$, Haythem Ali $^{2}$ \\ ${ }^{1}$ Faculty of Medicine, Department of Surgery, Beni-Suef University, Beni-Suef, Egypt \\ ${ }^{2}$ Maidstone and Tunbridge Wells Hospitals, Department of Surgery, Foundation Trust, UK \\ Email address: \\ mohamedsalah_2000@hotmail.com (M. S. Abdelhamid) \\ ${ }^{*}$ Corresponding author
}

\section{To cite this article:}

Mohamed Salah Abdelhamid, Ayman Abouleid, Ahmad Mohamed Sadat, Ahmad Hamouda, Amir Nisar, Haythem Ali. The Necessity of Staging Laparoscopy/Thoracoscopy in Patients Undergoing Thoracolaparoscopic Oesophagogastric Resections for Malignancy. Advances in Surgical Sciences. Vol. 4, No. 3, 2016, pp. 9-12. doi: 10.11648/j.ass.20160403.11

Received: March 22, 2016; Accepted: March 30, 2016; Published: April 27, 2016

\begin{abstract}
The benefit of thoracolaparoscopy in the staging algorithm for patients with upper gastrointestinal malignancies is considered to be valuable. The impact of modern staging modalities on the need for staging thoracolaparoscopy is unclear. Here we assess the possible role of diagnostic thoracolaparoscopy in preoperative staging of oesophagogastric cancer. No pure gastric lesion included in the study. We have included 102 cases of esophagogastric junction and 86 lower esophagus. Staging thoracolaparoscopy was carried out for 188 consecutive patients who were considered for curative oesophagogastric resection and had undergone complete set of staging invitation. In our study, 188 patients with oesophagogastric cancer had staging thoracolaparoscopy. The mean operative time was 43.9 minutes. Overall staging thoracolaparoscopy had changed treatment decision in $23 / 188$ patients $(12.2 \%)$ where no further curative resection was attempted due to involvement of peritoneum in 18 patients $(9.6 \%)$, omentum in 14patients (7.4\%), liver in 11 patients $(5.9 \%)$ and fixation of the stomach in 17 patients $(9 \%)$. We concluded that staging thoracolaparoscopy should be used for patients with esophageal cancer who are potential candidates for curative surgical resection based on a negative preoperative staging for lymph node or distant metastases Staging thoracolaparoscopy is very necessary and found to be safe and useful in detecting peritoneal, omental and liver disease despite negative staging modalities. No further curative resection was attempted due to involvement of the peritoneum, omentum, liver, and fixation of the stomach.
\end{abstract}

Keywords: Thoracolaparoscopy, Oesophagectomy, Staging

\section{Introduction}

The advent of videosurgery had moved us from the era of open to that minimally invasive surgery revolution [1].

The use of laparoscopic techniques has primarily occurred over the last two decades, the span of their development is 3 centuries [2].

The main advantage of diagnostic laparoscopy over traditional open laparotomy is reduced morbidity, decreased postoperative pain, and a shortened length of hospital stay. Diagnostic laparoscopy is useful for making a definitive clinical diagnosis whenever there is a diagnostic dilemma even after routine diagnostic workup, including patients who are hemodynamically stable and have nonspecific abdominal pain, with suspected intra-abdominal pathology, or critically ill intensive care unit patients with suspected intra-abdominal sepsis or pathologies [3].

Esophageal cancer often presents with locally advanced tumors, lymph node and/or distant metastases, and are associated with an overall poor prognosis. However, as with 
other gastrointestinal malignancies, preoperative imaging may suggest resectable disease, although a significant percentage of esophageal cancers $(20-65 \%)$ are found to be unresectable at the time of exploration. Diagnostic laparoscopy is particularly valuable in staging of esophageal cancer as it helps to identify patients who may or may not benefit from preoperative chemotherapy and thus avoid Laparotomy and/or Thoracotomy with negative findings [4].

Staging laparoscopy is useful in the evaluation in the following aspects: accurate staging of the tumor,

avoidance of non therapeutic intervention in patients with metastatic diseases, excludsion of metastatic disease or obtaining tissue biopsy prior to the initiation of neoadjuvant chemotherapy, obtaining tissue for diagnosis or performing peritoneal lavage cytology to exclude the presence of occult peritoneal metastasis, identification of patients with locally advanced disease (fixed tumor or vascular invasion) when there is no evidence of distant metastasis, selection of appropriate palliative treatment in patients with advanced or metastatic disease prior to definitive intervention after completion of neoadjuvant chemotherapy to assess treatment response or disease progression [5].

Absolute contraindications for staging laparoscopy include the following:

a- Known or obvious indications for therapeutic intervention as perforation or peritonitis.

b- Known intra-abdominal injury, complications of previous surgery, shock, evisceration, or abdominal wall dehiscence.

c- Acute intestinal obstruction associated with a massive bowel dilatation ( $>4 \mathrm{~cm}$ ), which may obscure the laparoscopic view and increase the likelihood of bowel injury.

d- Uncorrected coagulopathy.

e- Tense or distended abdomen (with suspected intraabdominal compartment syndrome).

f- Trauma with hemodynamic instability or a clear indication of bowel injuries, such as presence of bile or evisceration [6]

Relative contraindications for diagnostic laparoscopy include the following:

a- Intensive care unit patients who are too ill to tolerate pneumoperitoneum,

b- Potential hypercarbia or general anesthesia [7].

c- Presence of anterior abdominal wall infection (cellulitis or soft tissue infection).

d- Recent laparotomy (within 4-6 weeks) or extensive adhesions secondary to previous abdominal surgery.

e- Aortoiliac aneurysmal disease (may be associated with increased risk of vascular rupture).

f- Pregnancy (may be associated with injury to gravid uterus or fetal distress).

g- Cardiopulmonary compromise and morbid obesity [8]

Regarding thoracoscopy the major contraindications are related to the ability to perform the procedure. As long as no contraindication exists for the ability to insert instruments into the pleural space, it can be performed safely. Even when the lung is adherent to the chest wall, the use of transthoracic ultrasound by interventional pulmonologists can allow identification of safe areas to insert the trocar and pleuroscope.

A pleural separation of at least $10 \mathrm{~mm}$ is recommended to minimize injury to the lung. In patients with small effusions, a pneumothorax may need to be induced by cannulating the pleural space and asking the patient to inspire deeply while the catheter is open to the atmosphere. The presence of a pneumothorax can then be confirmed with either chest radiograph or thoracic ultrasound at the bedside. This procedure is limited by the ability of the patient to tolerate a pneumothorax. In patients who already have an effusion, the concern regarding tolerance of a pneumothorax is not as worrisome because an equal volume of fluid would be replaced by air.

The following relative contraindications may be corrected and accounted for:

- Refractory cough

- Hypoxia

- Coagulopathy

- Thrombocytopenia[9]

\section{Method}

All patients were subjected to (CBC, LFT, KFT, HIV and HCV screening, upper endoscopy with biopsy, CXR, CT chest and abdomen). No pure gastric lesions were included in the study. Cases included in the study were 102 cases of esophagogastric junction and 86 cases of lower esophagus lesions. Staging thoracolaparoscopy was carried out for 188 consecutive patients who were considered for curative oesophagogastric resection and had undergone complete set of staging invitation

The patients are placed in the supine position, and pneumoperitoneum is established. A 30-degree laparoscope is recommended for optimal visualization. Additional ports in the left upper quadrant and epigastric area can be placed as needed. Full inspection of the peritoneal cavity helps evaluating for peritoneal or liver metastases. If no distant metastases are discovered, then the left lateral lobe of the liver is elevated to expose the gastroesophageal junction, and the patient is placed in steep reverse Trendelenburg position. The tumor is inspected for extension into the surrounding area. Lymph nodes in the gastrohepatic ligament or celiac axis suspected to be malignant are biopsied. An optional laparoscopic feeding jejunostomy can be placed when neoadjuvant therapy is planned [9].

In addition, combined thoracoscopic/laparoscopic staging has been described to improve staging for esophageal cancer by increasing the number of positive lymph nodes identified compared with conventional staging (level II) Specifically for the thoracoscopic evaluation, the patient is in full, left lateral decubitus position with single-lung ventilation. Two to three thoracic trocars are placed, and the mediastinal pleura overlying the esophagus is incised to identify and biopsy lymph nodes as needed [10]. 


\section{Results}

188 patients with oesophagogastric cancer had staging thoracolaparoscopy The mean operative time was 43.9 minutes. Overall staging laparoscopy had changed treatment decision in $23 / 188$ patients $(12.2 \%)$ where no further curative resection was attempted due to involvement of peritoneum in 18 patients $(9.6 \%)$, omentum in 14 patients $(7.4 \%)$, liver in 11 patients $(5.9 \%)$ and fixation of the stomach in 17 patients (9\%). Most patients had more than a lesion detected upon staging laparoscopy. No positive finding was recorded during thoracoscopy.

Table 1. Demographic data.

\begin{tabular}{lll}
\hline Age and sex & No. Of patients & Percentage \\
\hline 40 years & 28 & $14.8 \%$ \\
40-50 years & 42 & $22.3 \%$ \\
$50-60$ years & 51 & $27.1 \%$ \\
60 years & 67 & $35.6 \%$ \\
Males & 131 & $69.6 \%$ \\
Females & 57 & $30.3 \%$ \\
\hline
\end{tabular}

Table 2. Intraoperative data.

\begin{tabular}{|c|c|c|}
\hline Operative events & Time and occurance & Mean and percent \\
\hline Operative time & $185-265 \mathrm{~min}$ & Mean $210 \mathrm{~min}$ \\
\hline Staging time & $55-80 \mathrm{~min}$ & Mean $65 \mathrm{~min}$ \\
\hline $\begin{array}{l}\text { Change in staging by } \\
\text { laparoscopy }\end{array}$ & 23 & $12.2 \%$ \\
\hline $\begin{array}{l}\text { Change in staging by } \\
\text { thoracoscopy }\end{array}$ & 0 & $0 \%$ \\
\hline Vascular injury & 0 & $0 \%$ \\
\hline Visceral injury & 0 & $0 \%$ \\
\hline
\end{tabular}

Table 3. Post operative findings.

\begin{tabular}{lll}
\hline Findings & No of patients and LOS & Percent and mean \\
\hline Chest infection & $12 / 165$ & $7.2 \%$ \\
Pulmonary embolism & $8 / 165$ & $4.8 \%$ \\
Leak & $6 / 165$ & $3.6 \%$ \\
LOS & $11-23$ dayes & Mean 13 \\
\hline
\end{tabular}

Table 4. Causes and number of plan change.

\begin{tabular}{lllll}
\hline $\begin{array}{l}\text { Causes of } \\
\text { plan change }\end{array}$ & $\begin{array}{l}\text { Peritoneal } \\
\text { involvment }\end{array}$ & $\begin{array}{l}\text { Omental } \\
\text { nodules }\end{array}$ & $\begin{array}{l}\text { Hepatic } \\
\text { metastasis }\end{array}$ & $\begin{array}{l}\text { Gastric } \\
\text { fixation }\end{array}$ \\
\hline Number & 18 & 14 & 11 & 17 \\
\hline
\end{tabular}

\section{Discussion}

Diagnostic thoracolaparoscopy is an extremely useful staging tool in patients with intra-abdominal cancers (esophageal, gastric). By enabling accurate staging, diagnostic thoracolaparoscopy permits patient selection for curative resection or a neoadjuvant chemotherapy while avoiding non therapeutic laparotomy, which is associated with a delay in initiation of chemotherapy. [4]

In our work we could detect 23 cases among 188 cases, 18 with peritoneal nodules, 14 with omental metastasis, 11 with hepatic secondaries and 17 cases with fixed stomach. All the diagnostic tools failed in detecting these lesions despite the highly advanced technology. Staging thoracolaparoscopy in this aspect was able to avoid unnecessary intervention, decreasing the risk that the patient could face, in case of faliure to detect such lethal lesion. From the financial point of view the treatment become cost effective.

We found that $12.2 \%$ of our case with lesions could not be diagnosed. This finding goes hand in hand with that of many who stated that a significant percentage of intra-abdominal cancers prove to be inoperable due to metastatic or locally advanced disease despite a preoperative workup that suggests a likely resectable disease [11,3, and 4]

Chua et al [12] stated that as with other gastrointestinal malignancies, preoperative imaging may suggest resectable disease, although a significant percentage of esophageal cancers $(20-65 \%)$ are found to be unresectable at the time of exploration. Diagnostic laparoscopy is particularly valuable in staging of esophageal cancer as it helps to identify patients who may or may not benefit from preoperative chemotherapy and to avoid interventions with negative findings. This goes hand in hand with our finding that led to change in the strategy of treatment from curative resection to palliative interventions.

From the financial point of view, this has become cost effective. Proponents for the routine use of SL cite the high incidence of imaging occult metastatic disease found during thoracolaparoscopic examination leads to avoidance of unnecessary operations and thus benefits patients. Proponents for the selective use of SL argue that when high quality imaging is used, only a small percentage of patients benefit from SL, and under these circumstances the procedure is not cost-effective [13, 14].

A significant percentage of intra-abdominal cancers prove to be inoperable due to metastatic or locally advanced disease despite a preoperative workup that suggests a likely resectable disease. Historically, these patients would have undergone morbid negative laparotomies with associated complications and resultant delay in the initiation of adjuvant or palliative chemotherapy. Diagnostic laparoscopy for accurate staging of intra-abdominal malignancies is referred to as staging laparoscopy, and is performed as a standard part of the staging workup for an increasing number of cancer subtypes [15].

In esophageal cancer, staging laparoscopy has a reported accuracy of $75-80 \%$ in identifying peritoneal metastasis with a staging sensitivity and specificity of $64 \%$ and $70 \%$ compared to ultrasound (40-50\%) and CT scan (45-60\%). The utility of diagnostic laparoscopy in esophageal cancer is shown to improve with the addition of laparoscopic ultrasound and video thoracoscopy [16].

When all preoperative imaging indicates no metastatic disease, SL with or without laparoscopic ultrasound has a sensitivity of $71 \%$ in finding peritoneal metastases, $78 \%$ for nodal metastases, and $86 \%$ for liver metastases (level II) [2]. This compares with ultrasound sensitivities of $14 \%, 11 \%$, $86 \%$, respectively, and CT scan sensitivities of $14 \%, 55 \%$, $71 \%$, respectively (level II) [2]. The accuracy has been reported to be $75-80 \%$ (level III) [3]. However, several 
reports indicate that only $0.08-10 \%$ of patients actually had a change in their management based on the results of laparoscopy [17], this is in agreement with our study in which $12.2 \%$ of our patients had lesions that could not be detected with other diagnostic tools and upon doing staging laparoscopy positive finding were there and the strategy of intervention had been changed. This was the same outcome in the work of Krasna et al [18].

\section{Conclusion}

Staging thoracolaparoscopy should be used for patients with esophageal cancer who are potential candidates for curative surgical resection based on a negative preoperative staging for lymph node or distant metastases. Staging thoracolaparoscopy is very necessary to avoid surgery in non operable cases. It was found to be safe and useful in detecting peritoneal, omental and liver disease despite negative staging modalities. There was no further curative resection attempted due to involvement of the peritoneum, omentum, liver, and fixation of the stomach

\section{References}

[1] Kooby DA. (2006) Laparoscopic surgery for cancer: historical, theoretical, and technical considerations. Oncology 20; 917-27.

[2] Stellato TA (1992) History of laparoscopic surgery. Surg Clin North Am; 72(5): 997-1002.

[3] Hori Y (2008) Diagnostic laparoscopy guidelines: This guideline was prepared by the SAGES Guidelines Committee and reviewed and approved by the Board of Governors of the Society of American Gastrointestinal and Endoscopic Surgeons (SAGES), Surg Endosc. 22(5); 1353-83.

[4] Chang L, Stefanidis D, Richardson WS, Earle DB, Fanelli RD (2009) The role of staging laparoscopy for intraabdominal cancers: an evidence-based review. Surg Endosc 23(2); 23141.

[5] Modlin IM, Kidd M, Lye KD (2004) From the lumen to the laparoscope. Arch Surg 139(10); 1110-26.

[6] Leake PA, Cardoso R, Seevaratnam (2011) A systematic review of the accuracy and indications for diagnostic laparoscopy prior to curative-intent resection of gastric cancer. Gastric Cancer 11.
[7] Peris A, Matano S, Manca G (2009). Bedside diagnostic laparoscopy to diagnose intraabdominal pathology in the intensive care unit. Crit Care. 13(1); R25.

[8] Richardson WS, Stefanidis D, Chang L, Earle DB, Fanelli $\mathrm{RD}(2009)$ The role of diagnostic laparoscopy for chronic abdominal conditions: an evidence-based review. Surg Endosc 23(9); 2073-7.

[9] Morino M, Pellegrino L, Castagna E, Farinella E, Mao P (2006) Acute nonspecific abdominal pain: A randomized, controlled trial comparing early laparoscopy versus clinical observation. Ann Surg 244(6); 881-6.

[10] Heath EI, Kaufman HS, Talamini MA (2000). The role of laparoscopy in preoperative staging of esophageal cancer. Surg Endo 14; 495-499.

[11] Cuschieri A (2001) Role of video-laparoscopy in the staging of intra-abdominal lymphomas and gastrointestinal cancer. Semin Surg Oncol. 20(2); 167-72.

[12] Chua YJ, Cunningham D (2007) The UK NCRI MAGIC trial of perioperative chemotherapy in resectable gastric cancer: implications for clinical practice. Ann Surg Oncol 14(10); 2687-90.

[13] Kim HJ, D'Angelica M, Hiotis SP, Shoup M, Weber SM (2007) Laparoscopic staging for liver, biliary,pancreas, and gastric cancer. Curr Probl Surg 44(4); 228-69.

[14] Henny CP, Hofland J. Laparoscopic surgery: pitfalls due to anesthesia, positioning, and pneumoperitoneum. Surg Endosc. 2005 Sep. 19(9): 1163-71.

[15] de Graaf GW, Ayantunde AA, Parsons SL, Duffy JP, Welch Nt (2007) The role of staging laparoscopy in oesophagogastric cancers. Eur J Surg Oncol. 33(8); 988-92.

[16] Maggio AQ, Reece-Smith AM, Tang TY, Sadat U, Walsh SR. (2008) Early laparoscopy versus active observation in acute abdominal pain: systematic review and meta-analysis. Int $J$ Surg 6(5); 400-3.

[17] Karateke F, Özdogan M, Özyazici S, Das K, Menekse E, Gülnerman YC (2013) The management of penetrating abdominal trauma by diagnostic laparoscopy: a prospective non-randomized study. Ulus Travma Acil Cerrahi Derg 19(1); 53-7.

[18] Krasna MJ, Jiao X, Mao YS (2002) Thoracosopy/laparoscopy in the staging of esophageal cancer. Surg Laparosc Endosc Percutan Tech 12; 213-218. 